\title{
Hankel matrix transforms and operators
}

\author{
Suliman Al-Homidan
}

Correspondence: homidan@kfupm edu.sa

Department of Mathematics and Statistics, King Fahd University of Petroleum and Minerals, Dhahran 31261, P. O. Box 119, Saudi Arabia

\begin{abstract}
Hankel operators and Hankel transforms are required in a number of applications. This article proves a number of theorems that efficiently and accurately approximates a function using Hankel transforms and Hankel sum. A characterization of the Hankel matrix sequences and Hankel matrix of semi-periodic and almost periodic sequences are also given. This article also introduces the concepts of almost periodic Hankel matrix, multiplicative Hankel matrix and normal almost periodic Hankel matrix. Applications to trigonometric sequences are given.
\end{abstract}

Keywords: almost periodic Hankel matrix, Hankel operators, Hankel transforms, semiperiodic sequences, Summability

\section{Introduction}

A Hankel matrix is a square matrix (finite or infinite), that is constant on each diagonal orthogonal to the main diagonal. Its $(n, m)$ th entry is a function of $n+m$. The most famous Hankel matrix is the Hilbert matrix, whose $(n, m)$ th entry is $1 /(n+m-1), n$, $m=1,2, \ldots$. For basic properties of the Hankel matrix, we refer to Horn and Johnson [1] and Iohvidov [2]. Interesting properties of the Hilbert matrix are discussed by Choi [3]. Hankel operators can be defined in several different ways and they admit different understanding. Such variety is important in applications, since in each case we can choose an understanding that is most suitable for the problem considered. The definition of Hankel operators will be given in the next section. For Hankel operators and their applications to approximation theory, prediction theory, and linear system theory, we refer to [4]. Relevence of the Hankel matrix to optimization problem can be found in [5-8]. Nehari [9] published the first article on general Hankel operators and its relation to Fourier coefficients. Beylkin and Monzón [10] have introduced approximation of a function by exponential sum using results of the Hankel matrix. Applications of Hankel transform can be found in circular symmetry, analysis of central potential scattering [11], solenoidal magnetic field [12], and medical computed tomography [13]. Hankel operators have many applications in, for example, control theory see [4] and the references therein.

In Section 2, we study order of approximation of a function by the $n$th Fourier series partial sum of a Hankel matrix transform. Section 3 is devoted to the characterization of Hankel matrix which induces an operator that maps one convergent sequence to another having the same or different limits. This section also contains characterization of Hankel matrix sequences, Hankel matrix semi-periodic sequences and almost periodic sequences. In Section 4, concepts of almost periodic Hankel matrix, multiplicative Hankel matrix,

(c) 2012 Al-Homidan; licensee Springer. This is an Open Access article distributed under the terms of the Creative Commons Attribution License (http://creativecommons.org/licenses/by/2.0), which permits unrestricted use, distribution, and reproduction in any medium, provided the original work is properly cited. 
normal almost periodic Hankel matrix and related methods of summability are introduced. Applications to trigonometric sequences are given.

\section{Approximation by Hankel matrix}

Let $H$ be an infinite Hankel matrix, that is,

$$
H=\left[\begin{array}{llllll}
h_{1} & h_{2} & h_{3} & \cdots & h_{i} & \cdots \\
h_{2} & h_{3} & h_{4} & \cdots & h_{i+1} & \cdots \\
\vdots & \vdots & \vdots & \ddots & \ddots & \vdots \\
h_{i} & h_{i+1} & \cdots & \cdots & h_{2 i-1} & \cdots \\
\vdots & \vdots & \vdots & \vdots & \vdots & \vdots
\end{array}\right]
$$

An operator $T$ mapping $x=\left(\xi_{0}, \xi_{1}, \xi_{2}, \ldots, \xi_{k} \ldots\right)$ to $y=\left(\eta_{0}, \eta_{1}, \eta_{2}, \eta_{3}, \ldots, \eta_{k} \ldots\right)$ where

$$
\eta_{k}=\sum_{n=0}^{\infty} h_{n+k} \xi_{n,} \quad k=0,1,2,3 \ldots
$$

is called the operator induced by the Hankel matrix $H . \eta_{m}$ is called the Hankel matrix mean or transform of the sequence $\left\{\xi_{n}\right\}$.

Let $X$ be a function space, say $H_{p}$ be the classical dyadic Hardy space or $B_{i p}^{\alpha}$ the classical Besov space. Let $S_{n}(x)=\frac{1}{2} a_{0}+\sum_{k=1}^{n}\left(a_{k} \cos k x+b_{k} \sin k x\right)$ be $n$th partial sum of the Fourier series of $f \in X$.

Definition 2.1 A Hankel matrix is called convex if $\Delta_{k}^{2} \geq 0$, where $\Delta_{k}=h_{n+k}-h_{n+k+1}$ and $\Delta_{k}^{2}=\Delta_{k}-\Delta_{k+1}$.

We study here the order of $\left\|f-\eta_{m}\right\|_{X}$ for $X=H_{p}$ and $X=B_{i p}^{\alpha}, 0<\alpha<1$. More precisely, we can prove the following theorem:

Theorem 2.2 Let $f(x)$ be periodic with period $2 \pi, f(x) \in H_{2}(-\pi, \pi)$ and let the Fourier series associated with $f(x)$ be $\frac{1}{2} a_{0}+\sum_{k=1}^{\infty}\left(a_{k} \cos k x+b_{k} \sin k x\right)$. Let $\sigma_{n}(f, x)=\sum_{k=0}^{n} h_{n+k} S_{k}(x)$ and $\sigma_{n}(f, x)=\sum_{k=0}^{n} h_{n+k} S_{k}(x)$, where $h_{n+k}$ is a convex Hankel matrix. Then $\sigma_{n}(f, x)-f(x)=O(\omega(\delta, t))$.

Proof. It can be verified that $\sigma_{n}(f, x)-f(x)=\frac{1}{\pi} \int_{0}^{\pi} \varphi_{x}(t) K_{n}(t) d t$, where $\phi_{x}(t)=f(x+$ $t)+f(x-t)-2 f(x)$ and $K_{n}(t)=\frac{1}{2}+\sum_{k=1}^{n} h_{n+k} \cos k t$ then

$$
\sigma_{n}(f, x)-f(x)=\frac{1}{\pi} \int_{0}^{\delta} \varphi_{x}(t) K_{n}(t) d t+\frac{1}{\pi} \int_{\delta}^{\pi} \varphi_{x}(t) K_{n}(t) d t
$$

Let $M_{n}(\delta)=\max _{\delta \leq t \leq \pi}\left|K_{n}(t)\right|$, for all $\delta>0$. It can be checked that there exists a decreasing function in $[0, \pi]$ such that $\left|K_{n}(t)\right| \leq K_{n}^{*}(t)$, and $\int_{0}^{\pi}\left|K_{n}^{*}(t)\right| d t<M$. Infact, it is not difficult to show that $K_{n}(t)$ itself satisfies these condition, $\int_{0}^{\pi}\left|K_{n}(t)\right| d t<M$. We have

$$
\left|\int_{0}^{\delta} \varphi_{x}(t) K_{n}(t) d t\right| \leq \int_{0}^{\delta}\left|\varphi_{x}(t)\right| K_{n}^{*}(t) d t=\left[\phi_{x}(t) K_{n}^{*}(t)\right]_{0}^{\delta}+\int_{0}^{\delta} \phi_{x}(t) d\left[-K_{n}^{*}(t)\right],
$$


where $\phi_{x}(t)=\int_{0}^{t}\left|\varphi_{x}(u)\right| d u=O(\omega(\delta, t))$. This gives us

$$
\int_{0}^{\delta} \varphi_{x}(t) K_{n}(t) d t=O(\omega(\delta, t)), \quad \text { for } \delta>0 .
$$

So we have

$$
\left|\int_{\delta}^{\pi} \varphi_{x}(t) K_{n}(t) d t\right| \leq M_{n}(\delta)=\max _{\delta \leq t \leq \pi}\left|K_{n}(t)\right| \leq \frac{1}{\sin ^{2} \frac{\delta}{2}} \sum_{k=0}^{n-1}\left|\Delta_{k}^{2}\right|+\left|\Delta_{k}\right| .
$$

The following relations hold $(n+1)\left|\Delta_{\nu}\right|<M_{1}$ and $\sum_{k=0}^{n-1}\left|\Delta_{n k}^{2}\right| \rightarrow 0$ as $n \rightarrow \infty$. In view of these relations, $\left|\int_{\delta}^{\pi} \varphi_{n}(t) K_{n}(t) d t\right|=O(1)$. Therefore, from 4 we have $\sigma_{n}(f, x)-f$ $(x)=O(w(\delta, t)$.

Corollary 2.3 If $f \in B_{i p^{\prime}}^{\alpha} 0<\alpha<1$, then $\sigma_{n}(x)-f(x)=O\left(\frac{1}{n^{\alpha}}\right)$

Proof. For $f \in B_{i p}^{\alpha}$, we have $O\left(\omega(\delta, t)=O\left(\delta^{\alpha}\right)\right.$ choosing $\delta=\frac{1}{n}$, we get $\sigma_{n}(f, x)-f(x)=$ $O\left(n^{-\alpha}\right)$ for all $0<\alpha<1$.

\section{Hankel matrix operators}

In this section, we consider the theory of infinite Hankel matrix transformations and summa-bility. A sequence $x=\left\{\xi_{0}, \xi_{1}, \ldots, \xi_{k}, \ldots\right\}$ is called summable with respect to a Hankel matrix $H$ if $\eta_{n} \rightarrow s$, where $\eta_{n}=\sum_{k=0}^{\infty} h_{n+k} \xi_{k}$. See for example, [14]. $H$ is called regular if $\eta_{n} \rightarrow s$ wherever $\xi_{k} \rightarrow s$.

Theorem 3.1 A Hankel matrix $H=\left\{h_{k, n}\right\}$ is regular if and only if

(i) $\lim _{n \rightarrow \infty} h_{n+k}=0$

(ii) $\lim _{n \rightarrow \infty} \sum_{k=1}^{\infty} h_{n+k}=1$

(iii) $\sup _{n} \sum_{k=1}^{\infty}\left|h_{n+k}\right| \leq M$

Proof. Let conditions (i)-(iii) be satisfied and $\xi_{k} \rightarrow s$. Condition (iii) implies that for each $n \in \mathbb{N}$, the series $\Sigma\left|h_{n+k}\right|$ converges. Since

$$
\sum h_{n+k} \xi_{k}=\sum h_{n+k}\left(\xi_{k}-s\right)+s \sum h_{n+k}
$$

it follows that $\Sigma h_{n+k}\left(\xi_{k}-s\right)$ and $s \Sigma h_{n+k}$ converges for every $n$. Hence, by (i)

$$
\left|\sum_{k=1}^{\infty} h_{n+k} \varepsilon_{k}\right| \leq \sum_{k=1}^{p}\left|h_{n+k} \varepsilon_{k}\right|+\sum_{k=p+1}^{\infty}\left|h_{n+k} \varepsilon_{k}\right| \leq \sup _{k}\left|\varepsilon_{k}\right| \sum_{k=1}^{p}\left|h_{n+k}\right|+M \sup _{k>p}\left|\varepsilon_{k}\right| .
$$

for any $p \in \mathbb{N}$, where $\varepsilon_{k}=\xi_{k}-s$. This implies that

$$
\lim \sup _{n}\left|\sum h_{n+k} \varepsilon_{k}\right| \leq M \sup _{k>p}\left|\varepsilon_{k}\right| .
$$

So, letting $p \rightarrow \infty$, we find that $\Sigma h_{n+k}\left(\xi_{k}-s\right) \rightarrow 0$, which implies $\eta_{n} \rightarrow s$. 
To prove the converse, let Hankel matrix $H$ be regular. Taking $x=\left(\xi_{1}, \xi_{2}, \ldots, \xi_{k}, \ldots\right)=$ $e_{k}$, we see that $\left|\Sigma h_{n+k}\right|=1$ implies $(i)$. Taking $x=(1,1, \ldots 1, \ldots)=e$, we get $\lim _{n \rightarrow \infty} \sum_{k=1}^{\infty} h_{n+k} e_{k}=1$, that is, (ii) holds. For (iii), since $H$ is regular and if we apply the Banach-Steinhan theorem and closed graph theorem, we have

$$
\sup _{n}\left|\sum h_{n+k} \xi_{k}\right| \leq\|H\| \sup _{k}\left|\xi_{k}\right|
$$

for all $\xi$. Now, if we choose any $n \in \mathbb{N}$ and $r \in \mathbb{N}$ and define $\xi_{k}=\left|h_{n+k}\right| / k_{n+k}$ for $1 \leq$ $k \leq r$, and $\xi_{k}=0$ for $k>r$, then from (5), we have $\sum_{k=1}^{r}\left|h_{n+k}\right| \leq\|H\|$. Since this inequality holds for every $n$ and letting $r \rightarrow \infty$ therefore (iii) is satisfied.

The following theorem can be proved similarly.

Theorem 3.2 If $\left\{\xi_{k}\right\}$ is convergent, then $\left\{\eta_{n}\right\}$ is convergent if and only if

(i) $\sup _{n} \sum_{k=1}^{\infty}\left|h_{n+k}\right|<\infty$,

(ii) for each $p \in \mathbb{N}$, there exists $\lim _{n} \sum_{k=p}^{\infty} h_{n+k}=h_{p}$.

Moreover, if (i) and (ii) hold and $\xi_{k} \rightarrow s$ as $k \rightarrow \infty$, then

$$
\lim _{n} \sum_{k=1}^{\infty} h_{n+k} \xi_{k}=s h_{1}+\sum_{k=1}^{\infty}\left(h_{k}-h_{k+1}\right)\left(\xi_{k}-s\right) .
$$

\section{Hankel matrix transform of trigonometric sequences}

First, we prove the following characterization of a Hankel matrix transform convergence of a trigonometric sequence.

Theorem 4.1 Let $f(x)$ be a function integrable in the sense of Lebesgue in $[0,2 \pi]$ and periodic with period $2 \pi$. Let $\frac{1}{2} a_{0}+\sum_{n=1}^{\infty}\left(a_{n} \cos n x+b_{n} \sin n x\right)$ be the Fourier series of $f$ $(x)$ and let $\sum_{n=1}^{\infty}\left(b_{n} \cos n x-a_{n} \sin n x\right)$ be its conjugate series. Let $H=\left\{h_{n+k}\right\}$ be a Hankel matrix. Then the Hankel matrix transform of the sequence $\sum_{n=1}^{\infty} h_{n+k} k\left(b_{k} \cos k x-a_{k} \sin k x\right)$, converges to $\pi^{-1} D(x)$ if and only if

$$
\lim _{n \rightarrow \infty} \sum_{k=0}^{n} h_{n+k} \cos k t=0
$$

in every $0<t \leq \pi$, where $a_{k}$ and $b_{k}$ are respectively, cosine and sine Fourier coefficients of a function of bounded variation on $[0,2 \pi]$, and $D(x)=f(x+0)-f(x-0)$.

Proof. Since

$$
\begin{aligned}
\sum_{k=0}^{n} h_{n+k} k\left(b_{k} \cos k x-a_{k} \sin k x\right) & =\sum_{k=0}^{n} h_{n+k} \frac{1}{\pi} \int_{0}^{\pi} \varphi_{x}(t) k \sin k t d t \\
& =\pi^{-1} D(x) \sum_{k=0}^{n} h_{n+k}+\pi^{-1} \int_{0}^{\pi} \sum_{k=0}^{n} h_{n+k} \cos k t d \varphi_{x}(t) \\
& =\pi^{-1} D(x)+\pi^{-1} \int_{0}^{\pi} K_{n}(t) d \varphi_{x}(t),
\end{aligned}
$$


where $\phi_{x}(t)=\phi_{x}(f ; t)=f(x+t)-f(x-t)$ and $K_{n}(t)=\sum_{k=0}^{n} h_{n+k} \cos k t$, we have to show that (6) holds. Therefore for every function of bounded variation on $[0,2 \pi]$ and for every $x$ in $[0,2, \pi]$

$$
\lim _{n \rightarrow \infty} \int_{0}^{\pi} K_{n}(t) d \varphi_{x}(t)=0
$$

and conversely. Condition (7) is equivalent to the following condition:

$$
\lim _{n \rightarrow \infty} \int_{\delta}^{\pi} K_{n}(t) d \varphi_{x}(t)=0
$$

for every function of bounded variation $f$ on $[0,2 \pi]$, for every $x$ in $[0,2 \pi]$, and every $0<\delta \leq \pi$. Suppose (7) holds. If $f$ is a function of bounded variation on $[0,2 \pi]$, and $x \in$ $[0,2 \pi]$, then for every $0<\delta \leq \pi$, we can construct a function of bounded variation $g$ on $[0,2 \pi]$ such that $g$ is constant in $[x-\delta, x+\delta]$ and $g$ coincides with $f$ elsewhere. Since

$$
\int_{\delta}^{\pi} K_{n}(t) d \varphi_{x}(f ; t)=\int_{0}^{\pi} K_{n}(t) d \varphi_{x}(g ; t)
$$

and the right-hand integral tends to 0 as $n \rightarrow \infty$ by (7) so does the left integral. Thus (7) implies (8). Suppose now (8) holds. If $f$ is a function of bounded variation on [0, $2 \pi]$ and $x \in[0,2 \pi]$, given any $\varepsilon>0$, then there exists a $\delta>0$ such that

$$
\int_{0}^{\delta}\left|d \varphi_{x}(t)\right|<\varepsilon / 2 M
$$

where $M$ is the positive constant such that

$$
\sum_{k=0}^{n}\left|h_{n+k}\right| \leq M \text { and so }\left|\int_{0}^{\delta} K_{n}(t) d \varphi_{n}(t)\right|<\varepsilon / 2 .
$$

Since (8) holds, there exists an $n_{0}=n_{0}(\varepsilon)$ such that for $n \geq n_{0}$,

$$
\left|\int_{\delta}^{\pi} K_{n}(t) d \varphi_{x}(t)\right|<\varepsilon / 2
$$

Combining (9) and (10), we have for $n \geq n_{0},\left|\int_{0}^{\pi} K_{n}(t) d \varphi_{x}(t)\right|<\varepsilon$, that is, (7) holds. Thus, (8) implies (7). By a well known theorem on the weak convergence of sequences in the Banach space of all continuous functions defined in a finite closed interval, it follows that (8) holds if and only if $\left|K_{n}(t)\right| \leq K$ for all $n$ and for all $t \in[\delta, \pi]$ and (6) holds. Since $\left|K_{n}(t)\right| \leq K$ for all $n$ and or all $t \in[\delta, \pi]$ is automatically satised by $\sum_{k=0}^{n}\left|h_{n+k}\right| \leq M$ independently of $n$, it follows that, (8) holds if and only if (6) holds. This ends the proof.

Vermes [15] proved that an arbitrary matrix $\mathrm{H}$ sums every periodic sequence if and only if for every rational $t, \sum_{k=0}^{\infty} h_{n+k} e^{2 \pi i k t}$ converges and $\lim _{n \rightarrow \infty} \sum_{k=0}^{\infty} h_{n+k} e^{2 \pi i k t}$ exists. 
Definition 4.2 A sequence $\left\{x_{k}\right\}$ is called semi-periodic if for every $\varepsilon>0$, there exists an integer $r$ such that $\left|x_{k}-x_{k+r n}\right|<\varepsilon$ for every $n$ and $k$.

Let $P$ be the set of all periodic sequences of complex numbers and $S P$ is the closure of $P$. Berg et al. [16] proved that $S P$ is the set of all semi-periodic sequences. $P$ is a linear subspace of $\ell_{\infty}$.

Theorem 4.3 For an infinite Hankel matrix $H=\left\{h_{n+k}\right\}$, the $H$ transform of every semi-periodic sequence is convergent if and only if

(i) $\|H\|=\sup _{n \geq 0} \sum_{k=0}^{\infty} h_{n+k}<\infty$

(ii) $\lim _{n \rightarrow \infty} \sum_{k=0}^{\infty} h_{n+k} e^{2 \pi i k t}$ exists for all rational t.

Proof. If $\left\{x_{k}\right\} \in S P$, then for any $\varepsilon>0$, there exists a $\left\{y_{k}\right\} \in P$ such that $\left\|\left\{x_{k}\right\}-\left\{y_{k}\right\}\right\|_{\infty}<\varepsilon$. If $\left\{y_{k}\right\}$ is of period $r$, then there exist $r$ constants $\lambda_{1}, \ldots, \lambda_{\mathrm{r}}$ such that

$$
\sum_{\mu=1}^{r} e^{2 \pi i k \mu / r} \lambda_{\mu}=y_{k}, \quad k=0, \ldots, r-1
$$

so that

$$
\begin{aligned}
\left|\sum_{k=0}^{\infty} h_{m+k} x_{k}-\sum_{k=0}^{\infty} h_{n+k} y_{k}\right| & \leq\left|\sum_{k=0}^{\infty}\left(h_{m+k}-h_{n+k}\right)\left(x_{k}-y_{k}\right)\right| \\
& +\left|\sum_{k=0}^{\infty}\left(h_{m+k}-h_{n+k}\right)\left(\sum_{k=0}^{r} e^{2 \pi i k \mu / r} \lambda_{\mu}\right)\right| \\
& \leq 2\|H\| \varepsilon+\varepsilon
\end{aligned}
$$

for $n$ and $m$ sufficient large. Hence, $\lim _{n \rightarrow \infty} \sum_{k=0}^{\infty} h_{n+k} x_{k}$ exists. The converse can be established as in Berg et al. [16], and (ii) is immediate since $e^{2 \pi i k t}$ is periodic when $t$ is rational.

Definition 4.4 A sequence $\left\{x_{k}\right\}$ of complex numbers is called almost periodic if to any $\varepsilon>0$, there corresponds an integer $N=N(\varepsilon)>0$ such that, among any $N$ consecutive integers, there exists an integer $r$ with property $\left|x_{k}-x_{k+r}\right|<\varepsilon$ for all $k$. Let AP be the set of all almost periodic sequences of complex numbers.

$A P$ is a linear subspace of $\ell_{\infty}$ and $P \subset \bar{P}=S P \subset A P \subset \ell_{\infty}$. For $t$ irrational $\left\{e^{2 \pi i k t}\right\}$ is almost periodic but not semi-periodic.

Theorem 4.5 For infinite Hankel matrix $H=\left\{h_{n+k}\right\}$, the $H$ transform of an almost periodic sequences is convergent if and only if

(i) $\|H\|=\sup _{n \geq 0} \sum_{k=0}^{\infty}\left|h_{n+k}\right|<\infty$

(ii) $\lim _{n \rightarrow \infty} \sum_{k=0}^{\infty} h_{n+k} e^{2 \pi i k t}$ exist for all $t$.

Proof. Let $\sum_{k=0}^{n} h_{n+k} x_{k} \rightarrow s$ as $\rightarrow \infty$ for every almost periodic sequence $\left\{x_{k}\right\}$. Since for each $t,\left\{e^{2 \pi i k t}\right\}$ is almost periodic therefore (ii) holds. It can be checked that if $y \in \ell_{1}$, 
then $\left\|y_{1}\right\|_{\ell_{1}}$ is equal to the norm of $y$ on the Banach space of almost periodic sequences denoted by $\|y\|_{A P}$. For each fixed $n$, first

$$
y_{N}(x)=\sum_{k=0}^{N} h_{n+k} x_{k}
$$

where $x \in A P, y_{N}(x) \in A P$ and $\lim _{N \rightarrow \infty} y_{N}(x)$ exists for each $x \in A P$. By the uniform bounded principle,

$$
\left\|y_{n}\right\|_{A P}=\left\|y_{n}\right\|_{\ell_{1}}=\sum_{1}^{N}\left|h_{n+k}\right| \leq M_{n}<\infty .
$$

For each $N$ so that $\sum_{k=0}^{\infty}\left|h_{n+k}\right|<\infty$ for each $n$. If we put $z_{n}(x)=\sum_{k=0}^{\infty} h_{n+k} x_{k}, x \in$ $A P$, then $z_{n} \in A P$ and $\lim _{n \rightarrow \infty} z_{n}(x)$ exists for each $x \in A P$. Applying once more the uniform boundedness principle, we get

$$
\|H\|=\sup _{n \geq 0} \sum_{k=0}^{\infty}\left|h_{n+k}\right|<\infty .
$$

Thus (i) holds. To prove the sufficiency of conditions ( $i$ ) and (ii), we note that if $x=$ $\left\{x_{k}\right\} \in A P$, then there exists a sequence $\left\{\sum_{j=0}^{N} b_{j} e^{2 \pi i \lambda_{j} k}\right\} \in A P$, such that for all $k\left|x_{k}-\sum_{j=0}^{N} b_{j} e^{2 \pi i \lambda_{j} k}\right|<\varepsilon$. Now

$$
\begin{aligned}
\left|\sum_{k=0}^{\infty} h_{m+k} x_{k}-\sum_{k=0}^{\infty} h_{n+k} x_{k}\right| & \leq\left|\sum_{k=0}^{\infty}\left(h_{m, k}-h_{n+k}\right)\left(x_{k}-\sum_{j=0}^{N} b_{j} e^{2 \pi i \lambda_{j} k}\right)\right| \\
& +\left|\sum_{k=0}^{\infty}\left(h_{m+k}-h_{n+k}\right) \sum_{j=0}^{N} b_{j} e^{2 \pi i \lambda_{j} k}\right| \\
& \leq 2\|H\| \varepsilon+\varepsilon
\end{aligned}
$$

for $m$ and $n$ sufficiently large. Thus, $\lim _{n \rightarrow \infty} \sum_{k=0}^{\infty} h_{n+k} x_{k}$ exists.

Definition 4.6 A Hankel matrix $H=\left\{h_{n+k}\right\}$ is called a normal almost periodic if (i) $\lim _{n \rightarrow \infty} \sum_{k=0}^{\infty} h_{n+k}=1$ and (ii) $\lim _{n \rightarrow \infty} \sum_{k=0}^{\infty} h_{n+k} e^{2 \pi i k t}=0$ for all $t \in(0,1)$.

Every normal almost periodic Hankel matrix is normal.

Theorem 4.7 Let $H=\left\{h_{n+k}\right\}$ be a Hankel matrix with $\|H\|<\infty$. Then for every function of bounded variation $f$ on $[0,2 \pi]$ and for every $x \in[0,2 \pi]$, the Hankel transform of the sequence $\left\{C_{k} e^{i k x}\right\}$ is convergent to $(2 \pi)^{-1} D(x)$ if and only if $H$ is a normal almost periodic matrix, where $D(x)=F(x+0)-F(x-0)$.

Proof. The condition is necessary, for if we choose $F: F(t)=2 \pi$ for $0<t \leq 2 \pi$ and $F$ $(0)=0$, then $C_{k}=1$ for all $k, D(0)=2 \pi$ and $D(x)=0$ for $0<x<2 \pi$ so that $\lim _{k \rightarrow \infty} \sum_{k=0}^{\infty} h_{n+k} e^{2 \pi i k x}=0$ for all $x \in(0,1)$ and $\lim _{k \rightarrow \infty} \sum_{k=0}^{\infty} h_{n+k}=1$. To prove the converse, let $H$ be a normal almost periodic matrix. Then 


$$
\begin{aligned}
\sum_{k=0}^{\infty} h_{n+k} C_{k} e^{i k x}=\sum_{k=0}^{\infty} h_{n+k} \frac{1}{2 \pi} \sum_{j=0}^{\infty} D\left(x_{j}\right) e^{i k\left(x-x_{j}\right)}\left(x-x_{j}\right) \\
+(2 \pi)^{-1} \int_{0}^{2 \pi} K_{n}\left(\frac{x-t}{2 \pi}\right) d F_{c}(t),
\end{aligned}
$$

where $k_{n}(t)=\sum_{k=0}^{\infty} h_{n+k} e^{2 \pi i k t},\left\{x_{j}\right\}$ are the points of jump of $F$ in $[0,2 \pi)$ and $F_{c}$ is the continuous part of $F$. Clearly, the first term on the right tends to $D(x) / 2 \pi$ as $n \rightarrow \infty$. The second term on the right hand side tends to 0 as $n \rightarrow \infty$, since, given $\varepsilon>0$, there exists a $\delta>0$ such that

$$
\int_{x-\delta}^{x+\delta} \mid d\left(F_{c}(t) \mid<\frac{\varepsilon}{2}\|H\|^{-1}\right.
$$

so that

$$
\left|\int_{x-\delta}^{x+\delta} K_{n}\left(\frac{x-t}{2 \pi}\right) d F_{c}(t)\right|<\varepsilon / 2
$$

and, by the bounded convergence theorem

$$
\left|\left(\int_{0}^{x-\delta}+\int_{x+\delta}^{2 x}\right) K_{n}\left(\frac{x-t}{2 \pi}\right) d F_{c}(t)\right|<\varepsilon / 2
$$

for large $n$. Thus, the Hankel transform of $\left\{C_{k} e^{i k x}\right\}$ converges to $D(x) / 2 \pi$.

\section{Acknowledgements}

The author is grateful to King Fahd University of Petroleum and Minerals for providing excellent research facilities.

\section{Competing interests}

The author declares that they have no competing interests.

Received: 4 February 2012 Accepted: 18 April 2012 Published: 18 April 2012

\section{References}

1. Horn, R, Johnson, C: Matrix Analysis. Cambridge University Press, Cambridge (1985)

2. Iohvidov, I: Hankel and Toeplitz matrices and forms. Birkhäuser, Boston, Mass (1982) (Algebraic theory, Translated from the Russian by G. Philip A. Thijsse, With an introduction by I. Gohberg)

3. Choi, M: Tricks or treats with the Hilbert matrix. Am Math Monthly. 90(5):301-312 (1983)

4. Peller, V: Hankel Operators and their Applications. Springer Monographs in Mathematics. Springer-Verlag, New York (2003)

5. Al-Homidan, S: Hybrid methods for approximating Hankel matrix. Numer Algorithms. 32(1):57-66 (2003)

6. Al-Homidan, S: Solving Hankel matrix approximation problem using semidefinite programming. J Comput Appl Math. 202(2):304-314 (2007)

7. Macinnes, C: The solution to a structured matrix approximation problem using Grass-mann coordinates. SIAM J Matrix Anal Appl 21(2):446-453 (1999). (electronic)

8. Suffridge, T, Hayden, T: Approximation by a Hermitian positive semidefinite Toeplitz matrix. SIAM J Matrix Anal Appl. 14(3):721-734 (1993)

9. Nehari, Z: On bounded bilinear forms. Ann of Math. 65(2):153-162 (1957)

10. Beylkin, G, Monzón, L: On approximation of functions by exponential sums. Appl Comput Harmon Anal. 19(1):17-48 (2005)

11. Sharafeddin, O, Bowen, H, Kouri, D, Hoffman, D: Numerical evaluation of spherical Bessel transforms via fast Fourier transforms. J Comput Phys. 100(2):294-296 (1992)

12. Nachamkin, J, Maggiore, C: A Fourier Bessel transform method for efficiently calculating the magnetic field of solenoids. J Comput Phys. 37(1):41-55 (1980)

13. Higgins, W, Munson, D: A hankel transform approach to tomographic image reconstruction. IEEE Trans Med Im. 7, $59-72(1988)$ 
14. Maddox, l: Elements of Functional Analysis. Cambridge University Press, Cambridge, 2 (1988)

15. Vermes, P: Infinite matrices summing every periodic sequence. Nederl Akad Wetensch Proc Ser A $58=$ Indag Math. 17, 627-633 (1955)

16. Berg, I, Wilansky, A: Periodic, almost periodic, and semiperiodic sequences. Michigan Math J. 9, 363-368 (1962)

doi:10.1186/1029-242X-2012-92

Cite this article as: Al-Homidan: Hankel matrix transforms and operators. Journal of Inequalities and Applications 2012 2012:92.

Submit your manuscript to a SpringerOpen ${ }^{\circ}$ journal and benefit from:

- Convenient online submission

- Rigorous peer review

- Immediate publication on acceptance

- Open access: articles freely available online

- High visibility within the field

- Retaining the copyright to your article

Submit your next manuscript at $\gg$ springeropen.com 